\title{
Safety and feasibility of countering neurological impairment by intravenous administration of autologous cord blood in cerebral palsy
}

\author{
Young-Ho Lee ${ }^{1,5+}$, Kyung Vin Choi ${ }^{1 \dagger}$, Jin Hwa Moon ${ }^{1}$, Hyun-Joo Jun ${ }^{1}$, Hye-Ryeong Kang ${ }^{1}$, Se-In Oh², \\ Hyung Sun Kim², Jang Soo Um², Mi Jung Kim², Yun Young Choi ${ }^{2}$, Young-Jun Lee ${ }^{4}$, Hee-Jin Kim ${ }^{5}$, Jong-Hwa Lee ${ }^{6}$, \\ Su Min Son ${ }^{7}$, Soo-Jin Choi ${ }^{8}$, Wonil $\mathrm{Oh}^{8}$ and Yoon-Sun Yang ${ }^{8}$
}

\begin{abstract}
Backgrounds: We conducted a pilot study of the infusion of intravenous autologous cord blood (CB) in children with cerebral palsy (CP) to assess the safety and feasibility of the procedure as well as its potential efficacy in countering neurological impairment.

Methods: Patients diagnosed with CP were enrolled in this study if their parents had elected to bank their CB at birth. Cryopreserved CB units were thawed and infused intravenously over 10 20 minutes. We assessed potential efficacy over 6 months by brain magnetic resonance imaging (MRI)-diffusion tensor imaging (DTI), brain perfusion single-photon emission computed tomography (SPECT), and various evaluation tools for motor and cognitive functions.

Results: Twenty patients received autologous CB infusion and were evaluated. The types of CP were as follows: 11 quadriplegics, 6 hemiplegics, and 3 diplegics. Infusion was generally well-tolerated, although 5 patients experienced temporary nausea, hemoglobinuria, or urticaria during intravenous infusion. Diverse neurological domains improved in 5 patients (25\%) as assessed with developmental evaluation tools as well as by fractional anisotropy values in brain MRI-DTI. The neurologic improvement occurred significantly in patients with diplegia or hemiplegia rather than quadriplegia.
\end{abstract}

Conclusions: Autologous CB infusion is safe and feasible, and has yielded potential benefits in children with CP.

Keywords: Cerebral palsy, Cord blood, Mononuclear cells, Cell therapy

\section{Backgrounds}

Cord blood $(\mathrm{CB})$ was introduced for the first time in humans to reconstitute the hematopoietic system in patient with Fanconi anemia [1]. Since the first cord blood transplantation (CBT), more than 20,000 CBTs have been reported worldwide and more than 400,000 $\mathrm{CB}$ units have been stored in more than $100 \mathrm{CB}$ banks [2]. The clinical use of $\mathrm{CB}$ has expanded into various areas such as inherited metabolic disorders. CBT for Hurler syndrome resulted in either stabilization or

\footnotetext{
* Correspondence: kimmjreh@hanyang.ac.kr

+ Contributed equally

${ }^{2}$ Department of Rehabilitation Medicine, Hanyang University Medical Center, Seoul, Korea

Full list of author information is available at the end of the article
}

improvement of neurocognitive function, and maintenance of new skills [3]. CBT for infantile Krabbe disease was highly effective if patients received transplants early in the course of the disease [4]. In such patients, CBT can prevent demyelination in the central and, often, the peripheral nervous system, extending life and improving overall quality of life. Kurtzberg, et al found that donor cells could enter the brain and induce remyelination and improvement in neurologic function in demyelinating diseases.

Cerebral palsy (CP) describes a group of permanent disorders of movement and posture limiting activity, due to non-progressive disturbances that occurred in the developing fetal or infant brain [5]. The ultimate goal of any therapy program for $\mathrm{CP}$ is to help children achieve

\section{C) Biomed Central}


their maximum potential in the motor, cognitive, and social realms. Despite a wide range of medical and surgical interventions in children with $\mathrm{CP}$, and at risk of $\mathrm{CP}$, there is no cure and significant variability in outcome, in part due to the heterogeneous nature of the underlying brain pathology.

Recently, the clinical application of $C B$ in regenerative medicine has expanded using mesenchymal stem cells (MSC) and mononuclear cells (MNC). Since CB contains hematopoietic stem cells as well as a mixture of multipotent stem cells, such as unrestricted somatic stem cells, mesenchymal stem cells, and endothelial colony-forming cells, CB has the ability to regenerate numerous tissue types and improve their function. The evidence that $\mathrm{CB}$ cells express neurotrophic factors and produce cytokines that may be partially responsible for the functional brain repair, has prompted investigation of the therapeutic use of $\mathrm{CB}$ in various neurologic diseases [6,7]. MSC and MNC have been administered intrathecally and intravenously in experimental and clinical trials for neurologic disorders [8-11]. However, there is no definitive evidence concerning the optimum route for cell therapy. Intravenous infusion of autologous $\mathrm{CB}$ MNC in children with $\mathrm{CP}$ represents a novel and safe challenge that may involve a quite different mechanism of action from previous treatment methods.

We have conducted a single arm pilot study of intravenous autologous $\mathrm{CB}$ MNC infusion in children with $\mathrm{CP}$ to assess the safety and feasibility of the procedure as well as any effect in improving neurological function.

\section{Methods \\ Patients}

The study was approved by our institutional research ethics committee, and written informed parental consent was obtained for all patients. Twenty young patients aged 2 10 years and diagnosed with CP due to various causes were enrolled since their parents had elected to bank their CB privately (Medipost Biomedical Research Institutes, Seoul, Korea) at birth; patients with epilepsy were excluded. The participants were not provided with any additional medication or rehabilitation programs and for ethical reasons there was no control group. The diagnoses of CP were based on history taking, routine laboratory tests, genetic and metabolic studies, chromosome analysis, and neurological examination. To confirm the diagnoses, 2 clinicians, a pediatrician and a physiatrist, examined the patients. The major signs that collectively led to the diagnosis of CP were delayed motor milestones, abnormal neurologic examination, persistence of primitive reflexes, and abnormal postural reactions.

\section{Study protocol}

Cryopreserved $\mathrm{CB}$ was delivered from the $\mathrm{CB}$ bank and infused intravenously over 10 20 minutes after thawing at the bedside, followed by administration of hydration fluid for 4 6 hours. To assess the short-term safety of $\mathrm{CB}$ infusion, we monitored vital signs and checked for symptoms such as fever, chills, nausea, and vomiting that could be caused by toxicity of the cryoprotectant and/or by lysis of red blood cells upon thawing the CB. The whole volume of thawed $C B$ was infused and its cellular content then analyzed to enable evaluation of the impact of cell dose on clinical improvement. To assess the long-term safety of $\mathrm{CB}$ infusion and to measure neurodevelopmental changes, all the $\mathrm{CP}$ patients were followed up in the departments of pediatrics and rehabilitation medicine on the 4 th, 8 th, 12 th, 24 th weeks after infusion of CB.

The neurodevelopmental tests were carried out each time along with measurements of vital signs, weight and height, in addition to a complete blood cell count, urinalysis, and blood chemistry. Comparative analyses of brain perfusion single-photon emission computed tomography (SPECT), and magnetic resonance imaging (MRI) with diffusion tensor imaging (DTI), were performed at 24 weeks after infusion of CB.

\section{Neurodevelopmental examinations}

Before and after the infusion of $\mathrm{CB}$, all the $\mathrm{CP}$ patients were assessed by comprehensive neurodevelopmental examinations including the Denver development screening test II (DDST - II) as a gross developmental screening, the pediatric evaluation of disability inventory (PEDI) as a detailed developmental assessment, the gross motor function classification system (GMFCS) for gross motor function staging, the gross motor function measure-88 (GMFM) as a detailed motor function test, the manual ability classification system (MACS) for fine motor staging, and the quality of upper extremity skill test (QUEST) for fine motor function testing. The results for each examination tool were evaluated by well-trained physical and occupational therapists, and therapeutic responses were comprehensively assessed by rehabilitation specialists.

\section{Imaging studies}

Brain perfusion SPECT, and conventional brain MRI-DTI were performed to evaluate functional changes of the brain. Fractional anisotropy (FA) values were obtained from the DTI data for 26 regions of interest (ROIs). All ROIs were set by the two pediatric neurologists, and all measurements were performed twice, and mean values used for analysis. We compared pre- and post-treatment FA values in the same patient. We also evaluated the correlation of FA value changes with neurodevelopmental changes. Brain perfusion SPECT using Tc-99 m ethyl cysteinate dimer was performed as a baseline study and as a follow-up study 24 weeks after infusion of $\mathrm{CB}$. Brain 
perfusion SPECT images were evaluated visually by a nuclear medicine physician, and by semiquantitative analysis using an asymmetry index calculated as the relative uptake ratios in ROIs drawn in the frontal, temporal, parietal, and occipital cortices, basal ganglia and thalamus of both hemispheres.

\section{Statistical Analysis}

In the analysis of FA values, a weighted kappa analysis was performed to measure inter-rater agreement. The Wilcoxon signed-rank test was used to compare differences of FA values pre- and post-treatment. In the comparative analysis of global outcome in the clinical characteristics and infused total nucleated cell (TNC) counts at 24 weeks after infusion, the variables were compared using Fisher's exact test and Wilcoxon ranksum test. $P$ values of $\leq 0.05$ were considered significant. All statistical analyses were performed with SPSS (version 15.0, SPSS Inc, Chicago, IL, USA) statistical software.

\section{Results}

Twenty patients ( 8 male, 12 female) received autologous CB infusion and were evaluated. Mean age and body weight at infusion were 55 (23 91) months and 13.9 $(7.2 \sim 21.4) \mathrm{kg}$, respectively. Possible causes of the CP were: 8 hypoxic ischemic encephalopathies, 1 streptococcal meningitis, 1 middle cerebral artery infarction, 1 polymicrogyria, and 9 unknown. Types of CP were: 11 quadriplegia, 6 hemiplegia, 3 diplegia.

TNC of $5.5 \pm 3.8(0.6 \sim 15.65) \times 10^{7} / \mathrm{kg}$ were infused. The viability of the cells before freezing was $95.4 \pm$ $3.5 \%$; it was not measured after thawing. Infusion was generally well-tolerated, even without premedication, although 3 patients experienced temporary nausea and hemoglobinuria, and another 2 patients experienced hemoglobinuria and urticaria, respectively, but these were easily controlled with peniramine or intravenous hydration. Although 14 of the 20 patients had increased scores in at least one of the 4 neurodevelopmental tests after $\mathrm{CB}$ infusion, only 5 patients (4 hemiplegia, 1 diplegia) showed more improvement in the neurodevelopmental tests than would normally be expected over a 6 month period. The neurodevelopmental improvements occurred significantly in patients with hemiplegia or diplegia rather than with quadriplegia $(p=0.008)$. Other variables, such as sex, age, body weight, infused TNC counts as well as possible causes of CP did not show any significant differences in global outcomes. Overall therapeutic responses according to clinical characteristics and infused TNC counts of CP patients are shown in Table 1.

In the analysis of FA values in MRI-DTI, significant differences between pre-treatment and post-treatment values were found in only 3 ROIs (right temporal, corpus callosum, and right periventricular white matter) out of 26 . In the 5 patients who showed comprehensive improvements in the neurodevelopmental tests, we examined these areas to analyze the clinical significance of the FA value changes. The differences of white matter integrity (FA values) in ROIs of 5 patients who showed clinical improvement after autologous $\mathrm{CB}$ infusion are presented in Table 2.

In the brain perfusion SPECT analysis, 2 (UPN 8,9) of the patients who experienced clinical improvement displayed improved perfusion. Both had mild forms of hemiplegia, and reduced perfusion in the left thalamus in the baseline SPECT studies, which increased in the SPECT images at 24 weeks. Six patients (UPN 6,12,13,14,15,16) showed decreased perfusion in the unilateral thalamus in the baseline SPECT images, and this expanded to the contralateral thalamus in the subsequent SPECT images. The rest of the patients $(n=12)$ showed no significant changes in cerebral perfusion state in SPECT images.

The detailed changes in the 5 patients in whom we detected neurological improvement after $\mathrm{CB}$ infusion are set out below.

\section{UPN 8 (F/71 months old)}

This child had functional hemiplegia and polymicrogyria on conventional brain MRI. One month after CB infusion, she improved on the personal-social, fine motor-adaptive, and language scales of DDST-II. She became able to dress herself and brush her teeth on the personal-social scale of DDST-II. Her score on the fine motor adaptive scale increased markedly in three months. She could make piles of 6 to 8 blocks, and was able to draw straight lines and circles. In GMFM, her balance while standing was enhanced as was her ability to jump over sticks and stand on one foot. In terms of PEDI-functional skills, she showed increased mobility and a slight improvement in social function. She also had slightly increased self-care, mobility, and social function in terms of problem solving, self-awareness and time orientation in the PEDI-caregiver assistant scale (Figure 1). In the analysis of MRI-DTI, we noted increments of FA values in the right temporal area, among the three significant areas. She displayed decreased perfusion in the left thalamus and right basal ganglia in the baseline SPECT study, and improved perfusion in these areas in subsequent brain perfusion SPECT images (Figure 2).

UPN 9 (M/43 months old)

This child was a preterm baby with hypoxic-ischemic encephalopathy, displaying weakness on his left side with periventricular leukomalacia. Fine motor-adaptive function such as circle drawing and personal-social functioning such as independent dressing improved 
Table 1 Therapeutic responses to autologous cord blood infusion according to clinical characteristics and infused TNC counts

\begin{tabular}{|c|c|c|c|c|c|c|c|c|c|c|c|c|}
\hline \multirow[t]{2}{*}{ UPN } & \multirow[t]{2}{*}{ Sex } & \multirow[t]{2}{*}{ Age (Mo) } & \multirow[t]{2}{*}{ BW (Kg) } & \multirow[t]{2}{*}{ TNC $(10 \wedge 7 / \mathrm{kg})$} & \multicolumn{3}{|c|}{ Dx } & \multicolumn{5}{|c|}{ Tx Response } \\
\hline & & & & & Type & GA & Possible Causes & DDST II & PEDI & GMFCS & MACS & Overall \\
\hline 1 & $M$ & 80 & 13 & 5.38 & Quad & Full & Unknown & $N$ & Y & $N$ & $N$ & $\mathrm{~N}$ \\
\hline 2 & $\mathrm{~F}$ & 24 & 10 & 6.72 & Quad & Full & HIE, PVL & N & N & Y & N & $N$ \\
\hline 3 & $\mathrm{~F}$ & 91 & 14 & 10.92 & Quad & Full & Unknown & $\mathrm{N}$ & Y & $N$ & $N$ & $N$ \\
\hline 4 & $F$ & 91 & 19.5 & 2.87 & Quad & Full & HIE, MAS & $N$ & N & $N$ & $N$ & $N$ \\
\hline 5 & $\mathrm{~F}$ & 82 & 18.1 & 4.36 & $\mathrm{Di}$ & Full & Unknown & N & Y & $N$ & N & $\mathrm{N}$ \\
\hline 6 & $\mathrm{~F}$ & 28 & 7.2 & 9.58 & Quad & Full & Unknown & $N$ & $N$ & $N$ & $N$ & $N$ \\
\hline 7 & M & 31 & 11.2 & 5.72 & Quad & Full & Strep meningitis & N & N & $N$ & $N$ & $N$ \\
\hline 8 & $\mathrm{~F}$ & 71 & 21.4 & 2.89 & Hemi & Full & Polymicrogyria & Y & Y & $N$ & $N$ & Y \\
\hline 9 & M & 43 & 16.4 & 0.6 & Hemi & Preterm & HIE, PVL & Y & Y & $\mathrm{N}$ & Y & Y \\
\hline 10 & $\mathrm{~F}$ & 75 & 9.4 & 10.63 & Quad & Full & HIE, MAS & $N$ & N & $N$ & N & $N$ \\
\hline 11 & $\mathrm{~F}$ & 53 & 15.3 & 5.88 & Hemi & Full & Unknown & N & Y & $N$ & N & Y \\
\hline 12 & $\mathrm{~F}$ & 40 & 12.7 & 2.44 & Hemi & Preterm & HIE, PVL & $N$ & Y & $N$ & Y & Y \\
\hline 13 & M & 29 & 10.8 & 6.85 & $\mathrm{Di}$ & Preterm & HIE, PVL & Y & Y & $\mathrm{N}$ & N & Y \\
\hline 14 & M & 67 & 15 & 5.26 & Quad & Full & Unknown & $N$ & N & $N$ & $N$ & $N$ \\
\hline 15 & M & 78 & 20 & 5.14 & Hemi & Full & HIE, ICH, Infarction & Y & Y & $N$ & N & N \\
\hline 16 & $\mathrm{~F}$ & 71 & 17.9 & 2.86 & Di & Full & Unknown & N & Y & $N$ & N & N \\
\hline 17 & $F$ & 58 & 11.6 & 15.65 & Quad & Preterm & HIE, PVL & $N$ & Y & $N$ & N & N \\
\hline 18 & M & 29 & 11.3 & 0.71 & Quad & Full & Unknown & $N$ & Y & $N$ & $N$ & $N$ \\
\hline 19 & $\mathrm{~F}$ & 30 & 11.5 & 3.29 & Hemi & Full & MCA infarction & N & N & $N$ & $N$ & $N$ \\
\hline 20 & M & 23 & 10.9 & 2.25 & Quad & Full & Unknown & $\mathrm{N}$ & Y & $N$ & $N$ & $\mathrm{~N}$ \\
\hline
\end{tabular}

UPN; unique patient number, BW; body weight, TNC; total nucleated cells, Dx; diagnosis, Tx; treatment, GA; gestational age, DDST-Il; Denver developmental screening test II, PEDI; pediatric evaluation of disability inventory, GMFCS; Gross motor function classification system, MACS; Manual ability classification system, Quad; quadriplegic, Di; diplegic, Hemi; hemiplegic, Full; full-term, HIE; hypoxic ischemic encephalopathy, PVL; periventricular leukomalacia, MAS; meconium aspiration pneumonia, Strep; streptococcal, ICH; intracranial hemorrhage, MCA; middle cerebral artery, N; no, $\mathrm{Y}$; yes

3 months after CB infusion, and this was followed at 6 months by improvements in brushing teeth and following the rules of games, as well as gross motor functions on the DDST-II scale. Walking, running, and standing abilities, along with total scores in the GMFM, increased consistently. In PEDI-functional skills, mobility, self-care, and social function remained stationary for 3 months after CB infusion. In PEDIcaregiver assistance, all factors increased slightly after 2 months (Figure 3). In the analysis of MRI-DTI, we noted an increment of FA value in the right periventricular white matter. Perfusion in left thalamus was reduced in the baseline SPECT images, but improved in subsequent SPECT images.

UPN 11 (F/53 months old)

This little girl was hemiplegic with no defined cause. Scores on the personal-social scale in DDST-II, in skills such as brushing teeth with assistance, undressing, using fork and spoon, and tidying toys, increased dramatically 1 month after $C B$ infusion, and fine motor-adaptive activities such as tapping an object with either hand, picking a plum from a bottle and piling up pairs of blocks began to increase after 2 months. Standing ability in GMFM increased after the second month, and there was a gradual increase in functional skills and caregiver assistance in PEDI selfcare activities such as using utensils, grooming, washing her face and bowel management, and in social functions including language comprehension and playing with toys (Figure 4). In the analysis of MRI-DTI, an increment of FA values in the right posterior periventricular white matter and right periventricular white matter was noted. Perfusion in the left posterior frontal and upper temporal cortexes, left basal ganglia, left thalamus and left cerebellum was reduced in the baseline SPECT images, and did not change significantly in subsequent SPECT images.

UPN 12 (F/40 months old)

This little girl was a preterm baby with hypoxicischemic encephalopathy, and displayed weakness on her left side with periventricular leukomalacia. All functions except gross motor function in DDST-II improved by 2 months after CB infusion. She could dress herself and follow the rules of games on the personal-social scale, and was also able to use building blocks, draw circles, drawing a person and distinguish two straight lines on the fine motor-adaptive scale. Language improved markedly after the first month. She became able to understand the identities of objects, prepositions and adjectives on the language 
Table 2 Differences of white matter integrity (FA values) of the 5 patients who showed neurodevelopmental improvement

\begin{tabular}{|c|c|c|c|c|c|c|c|c|c|c|c|}
\hline \multicolumn{2}{|l|}{ ROls } & \multicolumn{2}{|c|}{ UPN 8} & \multicolumn{2}{|c|}{ UPN 9} & \multicolumn{2}{|c|}{ UPN 11} & \multicolumn{2}{|c|}{ UPN 12} & \multicolumn{2}{|c|}{ UPN 13} \\
\hline & & Pre & Post & Pre & Post & Pre & Post & Pre & Post & Pre & Post \\
\hline \multirow[t]{2}{*}{ Temporal } & $\mathrm{TR} *$ & 0.431 & 0.451 & 0.438 & 0.421 & 0.425 & 0.445 & 0.463 & 0.464 & 0.416 & 0.430 \\
\hline & $\mathrm{TL}$ & 0.483 & 0.457 & 0.448 & 0.456 & 0.319 & 0.346 & 0.460 & 0.444 & 0.423 & 0.460 \\
\hline \multirow[t]{2}{*}{ Orbito-frontal } & $\mathrm{FR}$ & 0.411 & 0.465 & 0.470 & 0.378 & 0.359 & 0.386 & 0.421 & 0.438 & 0.466 & 0.728 \\
\hline & $\mathrm{FL}$ & 0.469 & 0.490 & 0.450 & 0.383 & 0.319 & 0.336 & 0.423 & 0.438 & 0.462 & 0.450 \\
\hline \multirow[t]{2}{*}{ Inferior frontal } & IFR & 0.453 & 0.446 & 0.444 & 0.447 & 0.397 & 0.370 & 0.404 & 0.437 & 0.443 & 0.474 \\
\hline & $\mathrm{IFL}$ & 0.506 & 0.515 & 0.494 & 0.383 & 0.320 & 0.334 & 0.457 & 0.464 & 0.452 & 0.454 \\
\hline \multirow[t]{4}{*}{ Internal capsule } & $\mathrm{ICR}$ & 0.489 & 0.499 & 0.498 & 0.570 & 0.501 & 0.534 & 0.476 & 0.498 & 0.467 & 0.465 \\
\hline & $\mathrm{ICL}$ & 0.527 & 0.520 & 0.493 & 0.504 & 0.336 & 0.328 & 0.496 & 0.496 & 0.478 & 0.454 \\
\hline & $C C^{*}$ & 0.534 & 0.538 & 0.493 & 0.487 & 0.421 & 0.427 & 0.443 & 0.509 & 0.481 & 0.447 \\
\hline & $S$ & 0.502 & 0.536 & 0.472 & 0.495 & 0.421 & 0.421 & 0.512 & 0.549 & 0.471 & 0.465 \\
\hline \multirow[t]{4}{*}{ Periventricular white matter } & AWMR & 0.374 & 0.366 & 0.363 & 0.300 & 0.363 & 0.369 & 0.363 & 0.307 & 0.373 & 0.332 \\
\hline & AWML & 0.423 & 0.385 & 0.375 & 0.315 & 0.264 & 0.228 & 0.349 & 0.371 & 0.332 & 0.378 \\
\hline & PWMR* & 0.479 & 0.479 & 0.400 & 0.425 & 0.426 & 0.481 & 0.407 & 0.415 & 0.398 & 0.408 \\
\hline & PWML & 0.488 & 0.484 & 0.476 & 0.442 & 0.314 & 0.392 & 0.459 & 0.444 & 0.414 & 0.410 \\
\hline \multirow[t]{2}{*}{ Occipital } & OR & 0.443 & 0.457 & 0.523 & 0.449 & 0.441 & 0.446 & 0.450 & 0.430 & 0.414 & 0.434 \\
\hline & $\mathrm{OL}$ & 0.483 & 0.510 & 0.488 & 0.395 & 0.284 & 0.271 & 0.536 & 0.509 & 0.441 & 0.391 \\
\hline \multirow[t]{2}{*}{ Sup frontal subcortex } & SFR & 0.475 & 0.447 & 0.484 & 0.444 & 0.346 & 0.330 & 0.345 & 0.431 & 0.452 & 0.433 \\
\hline & $\mathrm{SFL}$ & 0.455 & 0.460 & 0.443 & 0.360 & 0.308 & 0.334 & 0.399 & 0.401 & 0.452 & 0.440 \\
\hline \multirow[t]{2}{*}{ Middle frontal paravent WM } & MFPAR & 0.336 & 0.319 & 0.363 & 0.379 & 0.389 & 0.396 & 0.378 & 0.381 & 0.389 & 0.414 \\
\hline & MFPAL & 0.455 & 0.447 & 0.433 & 0.389 & 0.263 & 0.237 & 0.385 & 0.387 & 0.387 & 0.438 \\
\hline \multirow[t]{2}{*}{ Parietal perivent WM } & PPER & 0.468 & 0.451 & 0.447 & 0.440 & 0.466 & 0.504 & 0.446 & 0.479 & 0.435 & 0.445 \\
\hline & PPEL & 0.519 & 0.514 & 0.458 & 0.456 & 0.342 & 0.350 & 0.453 & 0.458 & 0.446 & 0.453 \\
\hline \multirow[t]{2}{*}{ Post parietal perivent WM } & PPPER & 0.467 & 0.507 & 0.405 & 0.467 & 0.477 & 0.495 & 0.492 & 0.488 & 0.391 & 0.476 \\
\hline & PPPEL & 0.465 & 0.466 & 0.464 & 0.427 & 0.284 & 0.284 & 0.452 & 0.525 & 0.408 & 0.372 \\
\hline \multirow[t]{2}{*}{ Post parietal paravent WM } & PPPAR & 0.445 & 0.497 & 0.336 & 0.412 & 0.480 & 0.477 & 0.433 & 0.473 & 0.425 & 0.508 \\
\hline & PPPAL & 0.441 & 0.443 & 0.381 & 0.359 & 0.273 & 0.288 & 0.448 & 0.422 & 0.374 & 0.425 \\
\hline
\end{tabular}

UPN; unique patient number, TR; right temporal, TL; left temporal, FR; right orbito-frontal, FL; left orbito-frontal, IFR; right inferior frontal, IFL; left inferior frontal, ICR; right internal capsule, ICL; left internal capsule, CC; corpus callosum, S; splenium, AWMR; right anterior periventricular white matter, AWML; left anterior periventricular white matter, PWMR; right posterior periventricular white matter, PWML; left posterior periventricular white matter, OR; right occipital, OL; left occipital, SFR; right superior frontal subcortex, SFL; left superior frontal subcortex, MFPAR; right anterior middle frontal paraventricular white matter, MFPAL; left anterior middle frontal paraventricular white matter, PPER; right parietal periventricular white matter, PPEL; left parietal periventricular white matter, PPPER; right posterior parietal periventricular white matter, PPPEL; left posterior parietal periventricular white matter, PPPAR; right posterior parietal paraventricular white matter, PPPAL; left posterior parietal paraventricular white matter $(*$ indicates the ROIs demonstrated significant differences between pre-treatment and posttreatment values out of 26 ROls.)

scale. Walking and running gradually improved on GMFM, and all tests showed a gradual increase in PEDI-functional skills. Self-care, mobility, and social function increased dramatically on the PEDI-caregiver assistance scale (Figure 5). In the analysis of MRI-DTI, increments of FA values in the right temporal and corpus callosum were noted. There was decreased perfusion in the bilateral anterior medial frontal cortex, and a suspicion of decreased perfusion in the bilateral thalamus on the baseline SPECT images, and this was followed by a definite decrease of perfusion in the bilateral thalamus on subsequent SPECT images.

UPN 13 (M/29 months old)

This male child was diplegic and preterm with periventricular leukomalacia. One month after CB infusion language improved, and fine motor-adaptive functions followed after the second month. After the third month, personal-social skills including ability to undress, dress, brush teeth with help, put on a shirt and say the names of friends improved in DDST-II. In GMFM, standing improved dramatically, along with improvements in crawling and kneeling, as well as walking and running, after the third month. He was able to attempt to stand on alternate feet. In PEDI, all scales increased gradually. Improvements were achieved in using utensils, maintaining self-hygiene, bathing, bowel and bladder management, and problem-solving ability and timeorientation also improved (Figure 6). In the analysis of MRI-DTI, an increment of FA values in the right temporal area was noted. There was reduced perfusion in the right thalamus in the baseline SPECT 

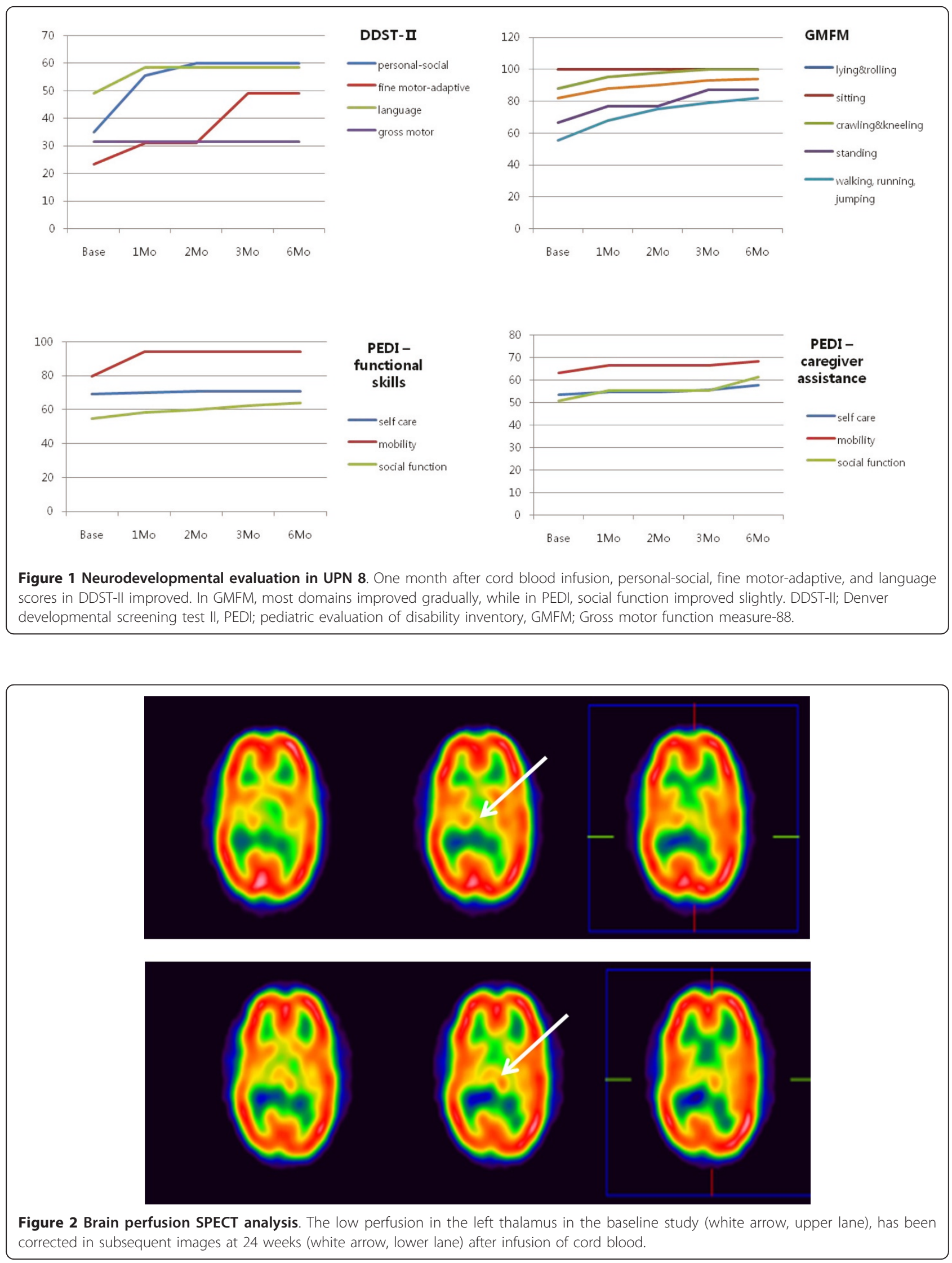

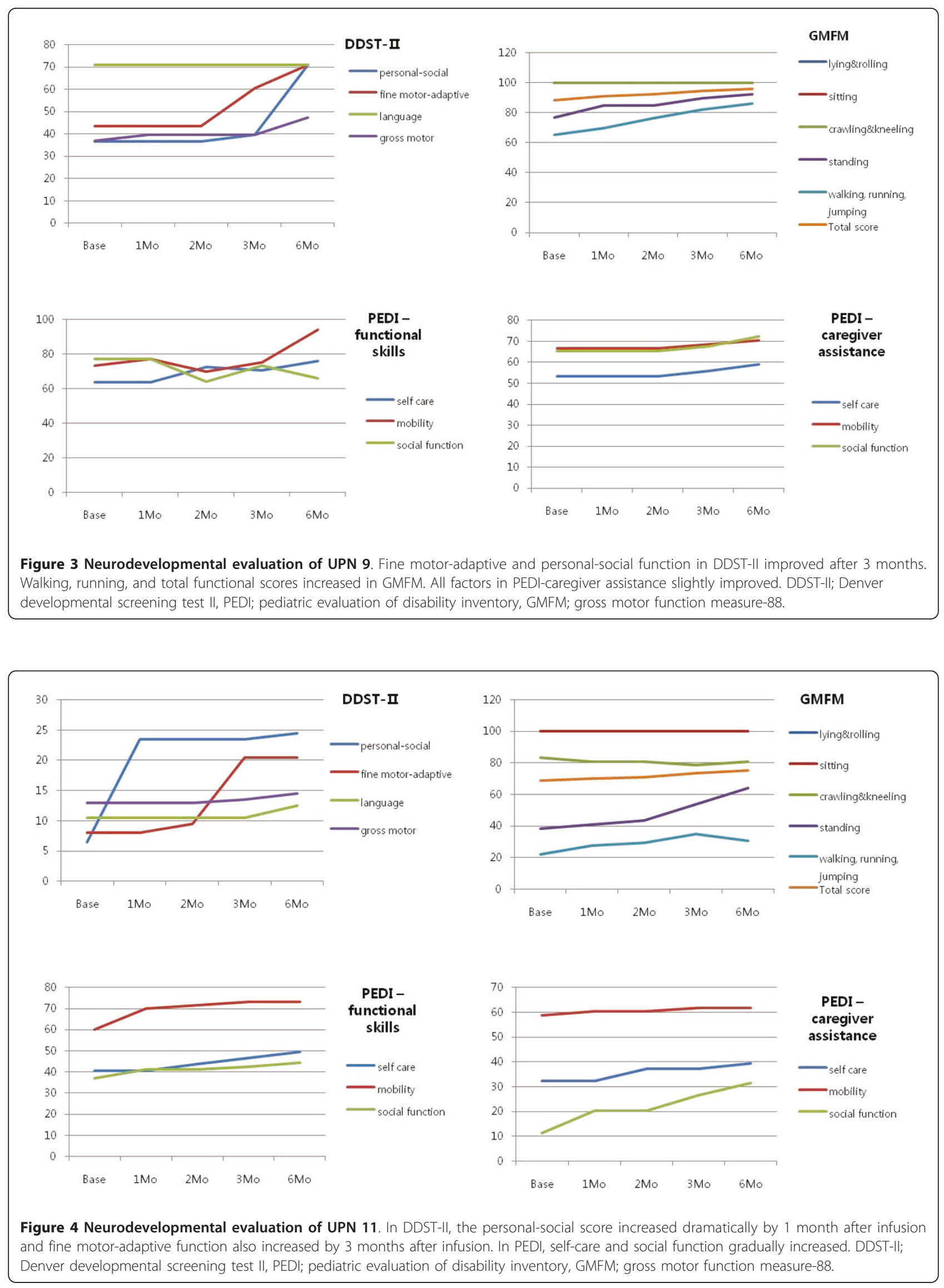


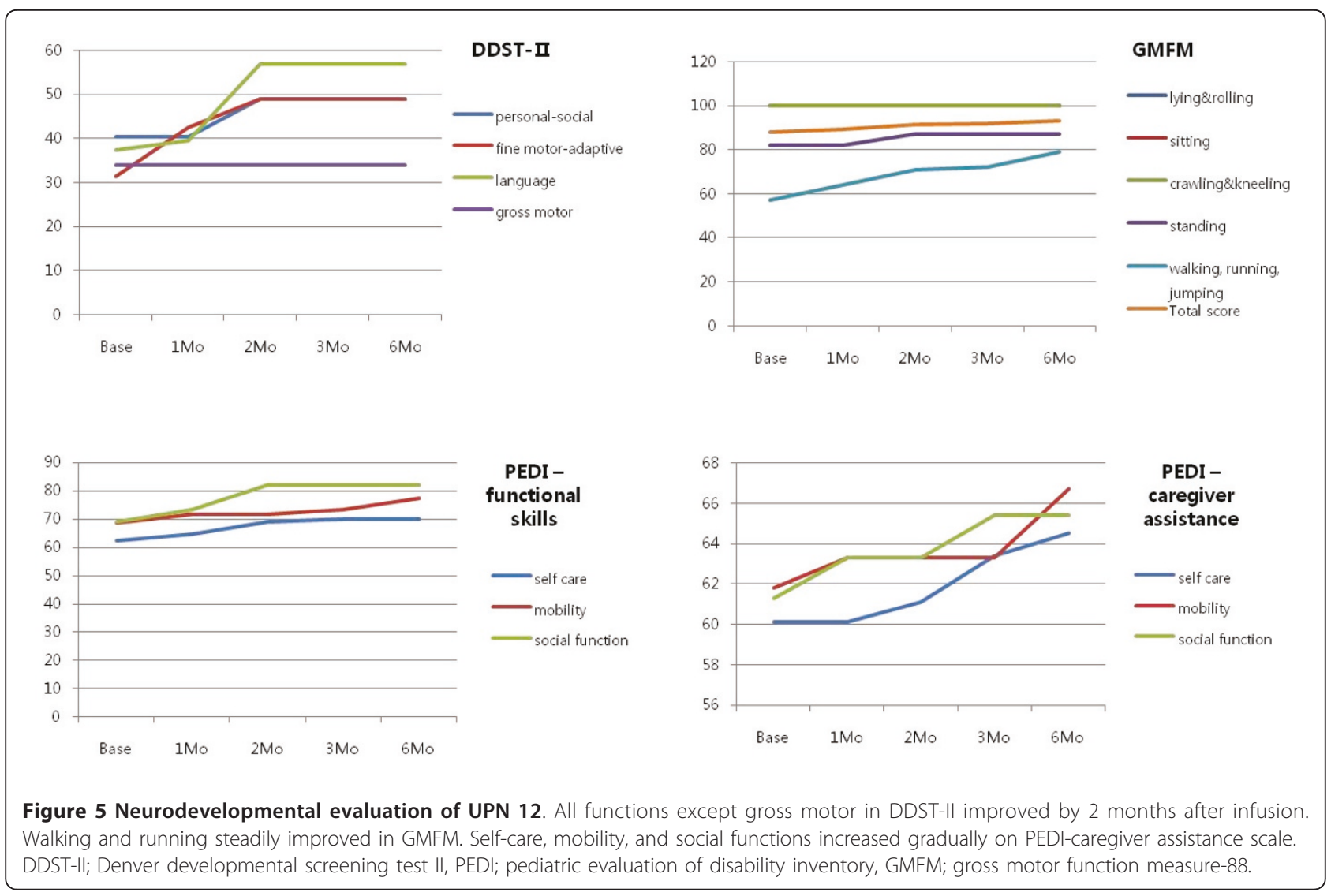

image, and perfusion worsened in the bilateral thalamus in the subsequent SPECT images.

\section{Discussion}

We observed that $25 \%$ of children with CP showed partial improvements in neurodevelopmental evaluation tests following intravenous, autologous CB MNC infusion. Interestingly, neurologic improvement was seen in patients with diplegia or hemiplegia rather than with quadriplegia, although this study is limited by the small number of subjects as well as the different types of $\mathrm{CP}$ and underlying causes. Brain plasticity is another limitation of this trial. However, we attempted to reduce any confounding effects of brain plasticity by including only patients between 2 and 10 years of age, to minimize the effect of brain plasticity during the first few years of life, and at the same time to maximize the kilogram-based cell dose of infused CB. We also demonstrated that age did not significantly affect global outcomes.

Abnormalities are demonstrable in many children with $\mathrm{CP}$ by conventional MRI, and DTI can be used to visualize brain white matter tracts, and has been shown to improve the detection of lesions in a range of neurologic disorders [12]. Trivedi, et al demonstrated a correlation between clinical grade and DTI measurements in motor and sensory pathways in children with CP [13]. Yoshida, et al demonstrated that ROI-based FA values in the corticospinal tract and posterior thalamic radiation were significantly lower in children with CP than in control children, and they suggested that the DTI parameters of corticospinal tract and posterior thalamic radiation are useful variables for evaluating clinical motor status and outcomes in children with CP [14]. Hoon, et al also showed a significant correlation between DTI scores in thalamocortical pathways and the quantitative clinical status of children with CP [15]. We also analyzed MRIDTI data to obtain imaging evidence of neurologic improvements. Based on findings from the aforementioned studies, we chose 24 weeks after CB infusion as the time for this analysis. Because we did not use control MRI-DTI data from healthy volunteers, we attempted to identify ROIs in which there were significant differences of FA values between pre- and post-treatment. We found that FA values in only 3 of the total of 26 ROIs displayed significant differences between pre- and post-treatment in the 19 evaluable patients. We assessed the correlation between clinical changes and changes in FA values in these 3 ROIs, in the 5 patients who showed neurodevelopmental improvements. The improved language scale and self awareness in UPN 8 may have been related to 


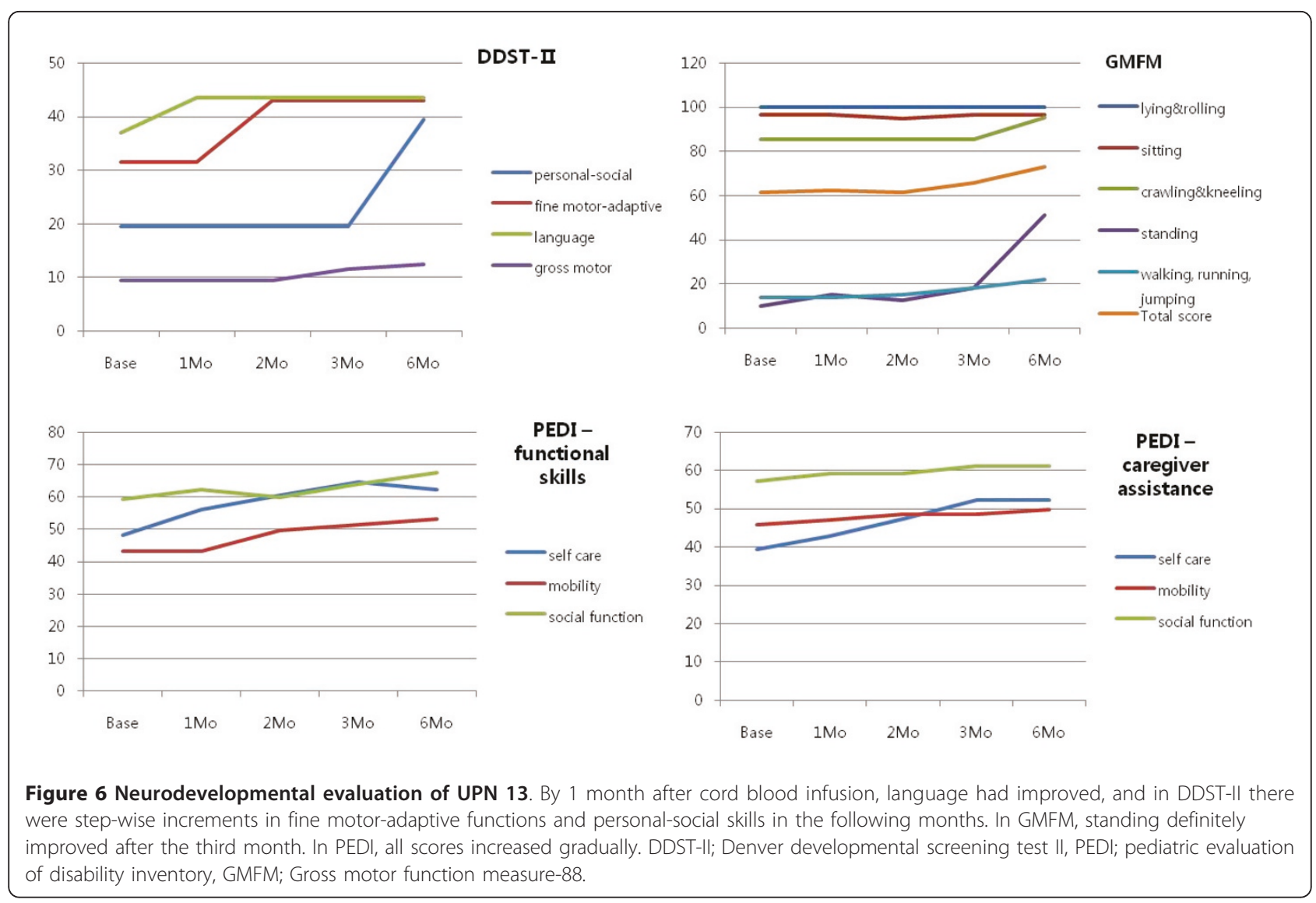

the increased FA values in the temporal lobe, but this latter cannot fully explain the overall improvements in the fine motor and social areas. FA values in the right periventricular white matter area increased in UPN 9 and could underlie his improvement in GMFM and fine motor-adaptive function. In UPN 11, the MRI-DTI changes involving partial increases in the anisotropy of the temporal lobe and posterior thalamic radiation could be related to her improved language function and increased score in mobility and self care ability. FA values in the corpus callosum increased in UPN 12; the corpus callosum is a highly dense pathway of white matter connecting right and left hemispheres, and the increase of FA after treatment could indicate improvement in the connectivity of the corpus callosum that permitted more effective transfer of information between the two sides of the brain. In UPN 13, the increase in FA values in the right temporal area might explain the improved functioning of the language area, but does not account for the increased fine motor function, mobility and other overall improvements.

In the brain perfusion SPECT analysis, 2 of the patients who showed clinical improvement displayed improved perfusion in thalamic areas, while regions of decreased perfusion, mainly in thalamic areas, expanded in another
6 patients. However, small, deep-seated structures such as the thalamus are susceptible to artifacts in SPECT images caused by attenuation of the low energy gamma rays emitted by these structures. Decreased image activity can be caused by increased attenuation, so that subtle changes can be artifactual. Further studies using brain positron emission tomography images generated by higher energy gamma rays could provide more reliable data on small, deep-seated structures.

Most studies of tissue regeneration have been performed by injecting MSC into target organ, although a variety of stem cell sources and routes of administration have been used in both animal models and humans. A double-blind, randomized, controlled trial revealed that bone marrow-derived MSC may be more effective than bone marrow-derived MNC in increasing lower limb perfusion and promoting foot ulcer healing in diabetic patients [16]. However, intravenously injected MNC obtained from the bone marrow of rats also induced functional recovery and decreased neurodegeneration after sensorimotor cortical ischemia in rats [17]. Reich, et al also showed that heterogeneous MNC and even CD133-depleted fractions have the ability not only to reduce apoptosis in neuronal cells but also to induce the maintenance of neuronal phenotypes [18]. 
The potential therapeutic benefits of human CB cells for treating injuries, diseases, and neurodegeneration are becoming increasingly recognized. The mechanisms underlying functional recovery remain to be clarified. Although Chen, et al observed that intravenously administered CB cells entered the brain, survived, migrated, and improved functional recovery after stroke [9], few $\mathrm{CB}$ cells are present in ischemic region compared to the number that were infused $[19,20]$. The small number of neural cells derived from the $\mathrm{CB}$ cells appears unable to explain the significant improvement resulting from cell transplantation. Kurtzberg observed neurological improvements in children with inborn errors of metabolism following intravenous infusion of $\mathrm{CB}$ in allogeneic CBTs, and detected the transplanted donor cells in recipients' brains [4]. Based on these experiences, she examined the effect of autologous CB infusion on neurological function in children with $\mathrm{CP}$ and again observed functional improvements [21].

However, in contrast to conventional hematopoietic stem cell transplantation with $\mathrm{CB}$, non-hematopoietic applications such as cardiovascular or neurological indications do not require permanent graft survival because the therapeutic activities of the $\mathrm{CB}$ are believed to be mediated in many cases by growth factor secretion $[22,23]$. Fan, et al found significantly higher levels of brain-derived neurotrophic factors (BDNF) and neurotrophin- $4 / 5$ in the culture supernatants of $\mathrm{CB}$ MNC than of peripheral blood MNC [6].

The neurologic improvement we observed in our study may be due to some migration of MNC into the brain and the consequent cellular effects as well as above-mentioned neurotrophic effects of the CB. Intravenously administered $\mathrm{CB}$ cells would not enter the brain in CP patients without some challenge such as chemo-radiotherapy which could induce permeability changes in the blood brain barrier (BBB). Most studies of intravenous infusion of MNC to restore neurologic function have been performed in acute stages of neurological impairment when the BBB is usually disrupted. However, since Newman, et al have demonstrated that interleukin (IL)-8, monocyte chemotactic protein (MCP)-1, and IL-1 $\alpha$ are consistently expressed by CB MNC regardless of culture conditions [7], the cytokines in $\mathrm{CB}$ which have been cited as pro-inflammatory mediators may be able to affect endothelial cells in such a way as to cause alterations in tight junction structure, the BBB, and leukocyte migration [24]. Microglia, which differentiate from monocytes, are major players in neurogenesis [25]. In inflammatory conditions, there can be both intrinsic proliferation of parenchymal microglia and substantial recruitment of monocytes [26]. Although studies have shown that inflammation and microglial activation can be detrimental to adult neurogenesis, their effects have turned out to be more complex and there is recent experimental evidence that they can be beneficial under some circumstances and support various steps in adult neurogenesis. It is conceivable that the early detrimental effects of microglia after acute injury can in some situations be converted into a supportive role during the chronic phase [27]. Microglia could then release neurotrophic factors such as BDNF and glial cell line-derived neurotrophic factor (GDNF), remove synapses from damaged neurons and influence the synaptic connectivity of newly-formed neurons [28].

\section{Conclusion}

Autologous intravenous CB MNC infusion seems to be practicable and safe and has yielded potential benefits in children with CP. Further clinical studies including randomized, cross-over, long-term follow-up trials, as well as basic research into the underlying mechanisms, are needed.

\section{Abbreviations}

CB: cord blood; CP: cerebral palsy; MRI: magnetic resonance imaging; DTI: diffusion tensor imaging; SPECT: single-photon emission computed tomography; CBT: cord blood transplantation; MSC: mesenchymal stem cells; MNC: mononuclear cells; TNC: total nucleated cell; DDST-II: Denver development screening test II; PEDI: pediatric evaluation of disability inventory; GMFCS: gross motor function classification system; GMFM: gross motor function measure-88; MACS: manual ability classification system; QUEST: quality of upper extremity skill test; FA: fractional anisotrophy; ROI: regions of interest; IL: interleukin; $\mathrm{MCP}$ : monocyte chemotactic protein; $\mathrm{BBB}$ : blood brain barrier.

\section{Acknowledgements}

This study was supported by a grant of the Korea Healthcare Technology R\&D Project (A101712), Ministry for Health \& Welfare, Republic of Korea. This work was supported by a grant from the Korea Science and Engineering Foundation (2010-0029508) through the MRC for Regulation of Stem Cell Behaviors at Hanyang University College of Medicine, Republic of Korea.

\section{Author details}

${ }^{1}$ Department of Pediatrics \& Cord Blood Clinic, Hanyang University Medical Center, Seoul, Korea. ${ }^{2}$ Department of Rehabilitation Medicine, Hanyang University Medical Center, Seoul, Korea. ${ }^{3}$ Department of Nuclear Medicine, Hanyang University Medical Center, Seoul, Korea. ${ }^{4}$ Department of Radiology, Hanyang University Medical Center, Seoul, Korea. ${ }^{5}$ Cell Therapy Center for Intractable Neurological Disorders, Hanyang University Medical Center, Seoul, Korea. ${ }^{6}$ Department of Pediatrics, Wonkwang University Medical Center, Sanbon, Korea. ${ }^{7}$ Department of Physical Medicine \& Rehabilitation, Yeungnam University Medical Center, Daegu, Korea. ${ }^{8}$ Medipost Biomedical Research Institute, Seoul, Korea.

\section{Authors' contributions}

YHL conceived and participated in the design of the study and drafted manuscript. KVC participated in its design and statistical analysis and also helped to draft the manuscript. JHM, JHL, YJL, HJK, YYC and SMS participated in the radiologic data analysis and interpretation. HSK, JSU, SIO and MJK participated in the evaluation and interpretation of neurologic functions. They also helped to draft manuscript. HJJ, HRK, SJC, WO and YSY participated in the coordination of patients and evaluations. All authors read and approved the final manuscript.

\section{Competing interests}

The authors declare that they have no competing interests.

Received: 18 January 2012 Accepted: 23 March 2012

Published: 23 March 2012 
References

1. Gluckman E, Broxmeyer HA, Auerbach AD, Friedman HS, Douglas GW, Devergie A, Esperou H, Thierry D, Socie G, Lehn P: Hematopoietic reconstitution in a patient with Fanconi's anemia by means of umbilicalcord blood from an HLA-identical sibling. N Engl J Med 1989, 321:1174-1178

2. Kurtzberg J: Update on umbilical cord blood transplantation. Curr Opin Pediatr 2009, 21:22-29.

3. Staba SL, Escolar ML, Poe M, Kim Y, Martin PL, Szabolcs P, Allison-Thacker J, Wood S, Wenger DA, Rubinstein P, Hopwood JJ, Krivit W, Kurtzberg J: Cordblood transplants from unrelated donors in patients with Hurler's syndrome. N Engl J Med 2004, 350:1960-1969.

4. Escolar ML, Poe MD, Provenzale JM, Richards KC, Allison J, Wood S, Wenger DA, Pietryga D, Wall D, Champagne M, Morse R, Krivit W, Kurtzberg J: Transplantation of umbilical-cord blood in babies with infantile Krabbe's disease. N Engl J Med 2005, 352(20):2069-2081.

5. Matsumoto T, Mugishima H: Non-hematopoietic stem cells in umbilical cord blood. Int I Stem Cell 2009, 2:83-89.

6. Fan CG, Zhang QJ, Tang FW, Han ZB, Wang GS, Han ZC: Human umbilical cord blood cells express neurotrophic factors. Neurosci let 2005, 380:322-325

7. Newman MB, Willing AE, Manresa JJ, Sanberg CD, Sanberg PR: Cytokines produced by cultured human umbilical cord blood cells: Implications for brain repair. Exp Neurol 2006, 199:201-208.

8. Joyce N, Annett G, Wirthlin L, Olson S, Bauer G, Nolta JA: Mesenchymal stem cells for the treatment of neurodegenerative disease. Regen Med 2010, 5:933-946.

9. Chen J, Sanberg PR, Li Y, Wang L, Lu M, Willing AE, Sanchez-Ramos J, Chopp M: Intravenous administration of human umbilical cord blood reduces behavioral deficits after stroke in rats. Stroke 2001, 32:2682-2688.

10. Papadopoulos KI, Low SSS, Aw TC, Chantarojanasiri T: Safety and feasibility of autologous umbilical cord blood transfusion in 2 toddlers with cerebral palsy and the role of low dose granulocyte-colony stimulating factor injections. Restor Neurol Neurosci 2011, 29:17-22.

11. Prabhakar S, Muthaian R, Chabbra R, Anand A: Analysis of homing potential of marrow-derived mononuclear cells in an experimentallyinduced brain stroke mouse model. Brain Inj 2010, 24:1485-1490.

12. Trivedi R, Gupta RK, Shah V, Tripathi M, Rathore RK, Kumar M, Pandey CM, Narayana PA: Treatment-induced plasticity in cerebral palsy: a diffusion tensor imaging study. Pediatr Neurol 2008, 39:341-349.

13. Trivedi R, Agarwal S, Shah V, Goyel P, Paliwal VK, Rathore RK, Gupta RK: Correlation of quantitative sensorimotor tractography with clinical grade of cerebral palsy. Neuroradiology 2010, 52:759-765.

14. Yoshida S, Hayakawa K, Yamamoto A, Okano S, Kanda T, Yamori Y Yoshida N, Hirota H: Quantitative diffusion tensor tractography of the motor and sensory tract in children with cerebral palsy. Dev Med Child Neurol 2010, 52:935-940.

15. Hoon AH Jr, Stashinko EE, Nagae LM, Lin DD, Keller J, Bastian A, Campbell ML, Levey E, Mori S, Johnston MV: Sensory and motor deficits in children with cerebral palsy born preterm correlate with diffusion tensor imaging abnormalities in thalamocortical pathways. Dev Med Child Neurol 2009, 51:697-704.

16. Lu D, Chen B, Liang Z, Deng W, Jiang Y, Li S, Xu J, Wu Q, Zhang Z, Xie B, Chen $S$ : Comparison of bone marrow mesenchymal stem cells with bone marrow-derived mononuclear cells for treatment of diabetic critical limb ischemia and foot ulcer: a double-blind, randomized, controlled trial. Diabetes Res Clin Pract 2011, 92:26-36.

17. Giraldi-Guimarães A, Rezende-Lima M, Bruno FP, Mendez-Otero R Treatment with bone marrow mononuclear cells induces functiona recovery and decreases neurodegeneration after sensorimotor cortical ischemia in rats. Brain Res 2009, 1266:108-120

18. Reich DM, Hau S, Stahl T, Scholz M, Naumann W, Emmrich F, Boltze J, Kamprad M: Neuronal hypoxia in vitro: investigation of therapeutic principles of HUCB-MNC and CD133+ stem cells. BMC Neurosci 2008, 9:91.

19. Vendrame M, Cassady CJ, Newcomb J, Bulter T, Pennypacker KR, Zigova T, Sanberg CD, Sanberg PR, Willing AE: Infusion of human umbilical cord blood cells in rat model of stroke dose-dependently rescues behavioral deficits and reduces infarct volume. Stroke 2004, 35(10):2390-2395.

20. Willing AE, Lixian J, Milliken M, Poulos S, Zigova T, Song S, Hart C, SanchezRamos J, Sanberg PR: Intravenous versus intrastriatal cord blood administration in a rodent model of stroke. J Neurosci Res 2003, 73:296-307.

21. Sun J, Allison J, McLaughlin C, Sledge L, Waters-Pick B, Wease S, Kurtzberg J: Differences in quality between privately and publicly banked umbilical cord blood units: a pilot study of autologous cord blood infusion in children with acquired neurologic disorders. Transfusion 2010, 50:1980-1987.

22. Bachstetter AD, Pabon MM, Cole MJ, Hudson CE, Sanberg PR, Willing AE, Bickford PC, Gemma C: Peripheral injection of human umbilical cord blood stimulates neurogenesis in the aged rat brain. BMC Neurosci 2008 14:9-22.

23. Xiao J, Nan Z, Motooka Y, Low WC: Transplantation of a novel cell line population of umbilical cord blood stem cells ameliorates neurological deficits associated with ischemic brain injury. Stem Cells Dev 2005, 14:722-733.

24. Stolp HB, Dziegielewska KM: Review: Role of developmental inflammation and blood-brain barrier dysfunction in neurodevelomental and neurodegenerative diseases. Neuropathol Appl Neurobiol 2009, 35:132-146.

25. Aloisi F: Immune function of microglia. Glia 2001, 36:165-179.

26. Flugel A, Bradl M, Kreutzberg GW, Graeber MB: Transformation of donorderived bone marrow precursors into host microglia during autoimmune CNS inflammation and during the retrograde response to axotomy. J Neurosci Res 2001, 66:74-82.

27. Ekdahl CT, Kokaia Z, Lindvall AO: Review: Brain inflammation and adult neurogenesis: The dual role of microglia. Neuroscience 2009, 158:1021-1029

28. Morgan SC, Taylor DL, Pocock JM: Microglia release activators of neuronal proliferation mediated by activation of mitogen-activated protein kinase, phosphatidylinositol-3-kinase/Akt and delta-Notch signalling cascades. $J$ Neurochem 2004, 90:89-101.

doi:10.1186/1479-5876-10-58

Cite this article as: Lee et al: Safety and feasibility of countering neurological impairment by intravenous administration of autologous cord blood in cerebral palsy. Journal of Translational Medicine 2012 10:58.

\section{Submit your next manuscript to BioMed Central and take full advantage of:}

- Convenient online submission

- Thorough peer review

- No space constraints or color figure charges

- Immediate publication on acceptance

- Inclusion in PubMed, CAS, Scopus and Google Scholar

- Research which is freely available for redistribution

Submit your manuscript at www.biomedcentral.com/submit
C Biomed Central 